\title{
Direct administration of zoledronate acid improves bone structure in local osteoporotic lesion of ovariectomized rats
}

Q

YOHEI MATSUO, M. Kashii, T. Sugiura, M. Furuya, T. Morimoto, H. Honda, T. Kaito, M. Iwasaki, H. Yoshikawa Dept. of Orthopedic Surgery, Osaka University Graduate School of Medicine, JAPAN Dept. of Orthopedic Surgry

\section{Introduction}

- Management of local osteoporotic lesions is a clinical difficult problem, and intravenous administration of bisphosphonates (BPs) has a limited effect for local osteoporotic or osteolytic lesion.

- Zoledronate acid (ZOL) is the most potent BP that strongly inhibits osteoclast function with high binding affinities for bone.

- We hypothesized that direct administration of high-dose ZOL for local osteoporotic or osteolytic lesion enables to improve local bone structure without any systemic side effects.

\section{Objectives}

To examine the efficacy and safety of direct administration of ZOL on local osteoporotic lesion of ovariectomized rats

\section{Materials \& Methods}

\section{Animals}

Rats Sprague Dawley (SD), 6 months-old, female (SLC, Japan)

\section{Regents}

Zoledronate acid (ZOL) purchased from Novartis Pharmaceuticals Japan

Dose: $\mathbf{6 7} \mathbf{\mu g} / \mathbf{k g}$ equal to the recommended dose for human

\section{Procedure of the local injection into the bone marrow}

1) Check the joint line using the scout view of micro CT (Fig.1)

2) Make the two holes at the right proximal tibia (Fig.2)

3) Flash and open the route between the two holes (Fig.3)

4) Administer ZOL or saline into the bone marrow

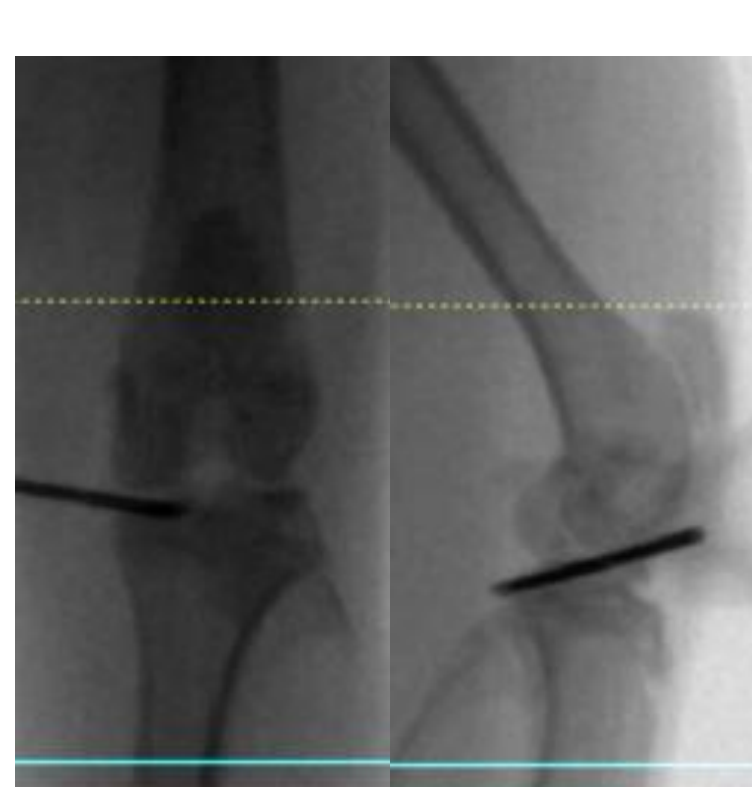

Fig.1

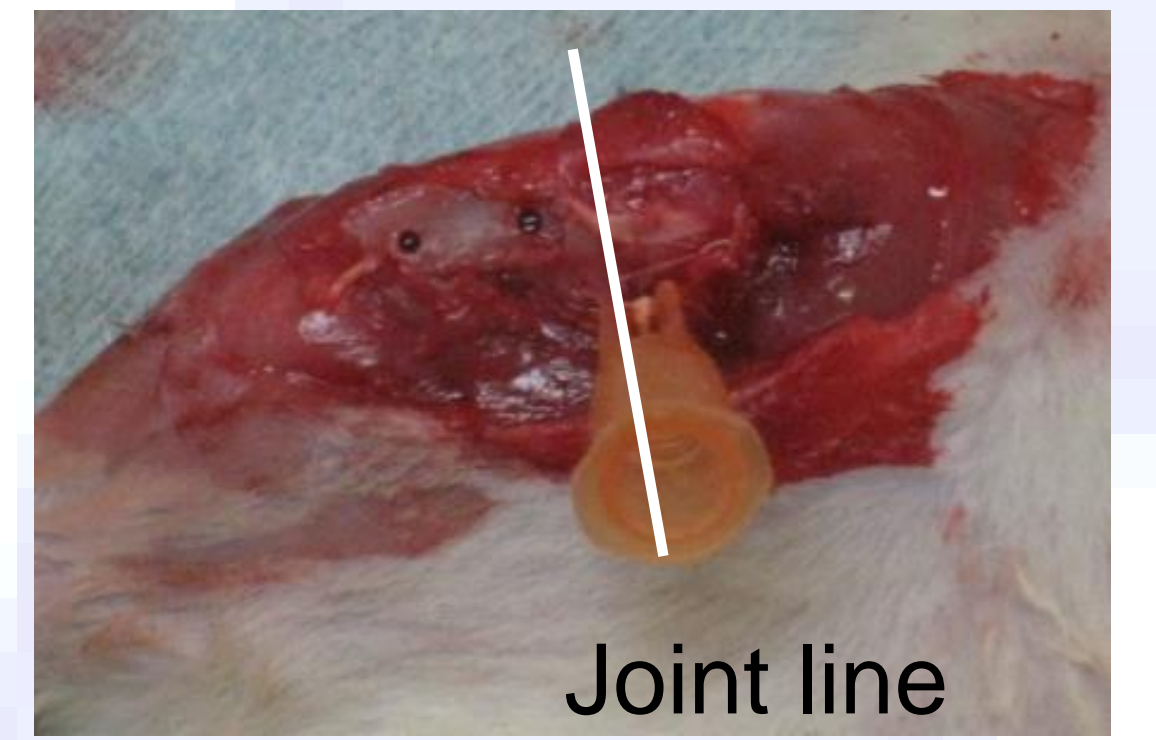

Fig.2

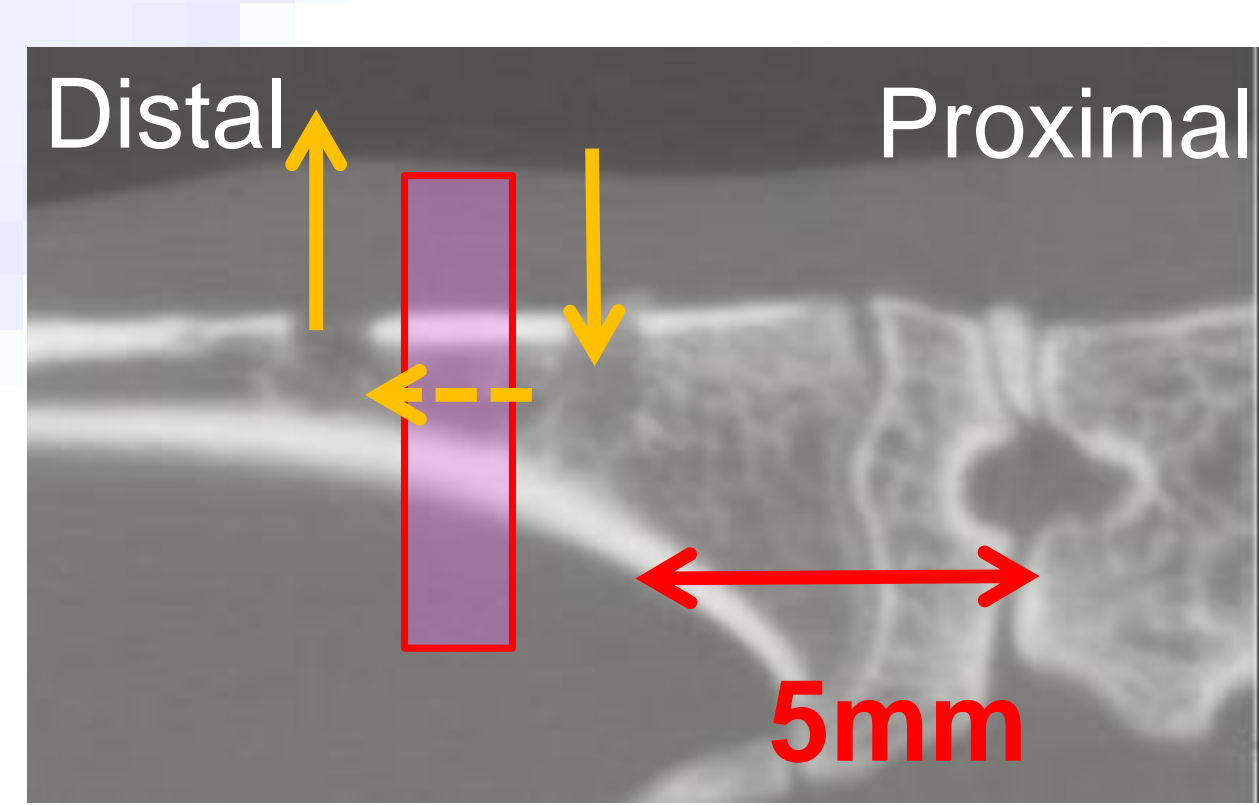

Fig.3

\section{Experimental design}

Ovariectomy (OVX) caused osteoporosis and deterioration of three-dimensional (3D) trabecular microstructure. Six weeks later after OVX, rats were divided into the two groups

\section{-6
OV
$\uparrow$}

2wks

4 wks

6 wks

$8 w k s$

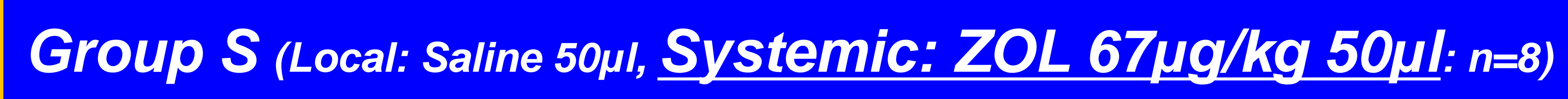

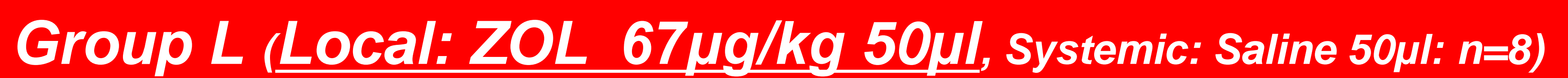
$\uparrow$

administration

(Local or Systemic)

Sacrifice

\section{Assessment}

\section{In vivo micro CT ( $\mu \mathrm{CT})$}

- Volumetric BMD measurements \& 3D trabecular microstructural analysis

- Measurement interval: 0, 2, 4, 6 and 8 weeks

- Analyzed area: local osteoporotic lesion

Area 1: Cancellous bone area of the right tibia between the two holes (Fig.3) Area 2: Mirror area of the left tibia

\section{Results}

Deleterious microstructure by OVX further improved in the group $L$
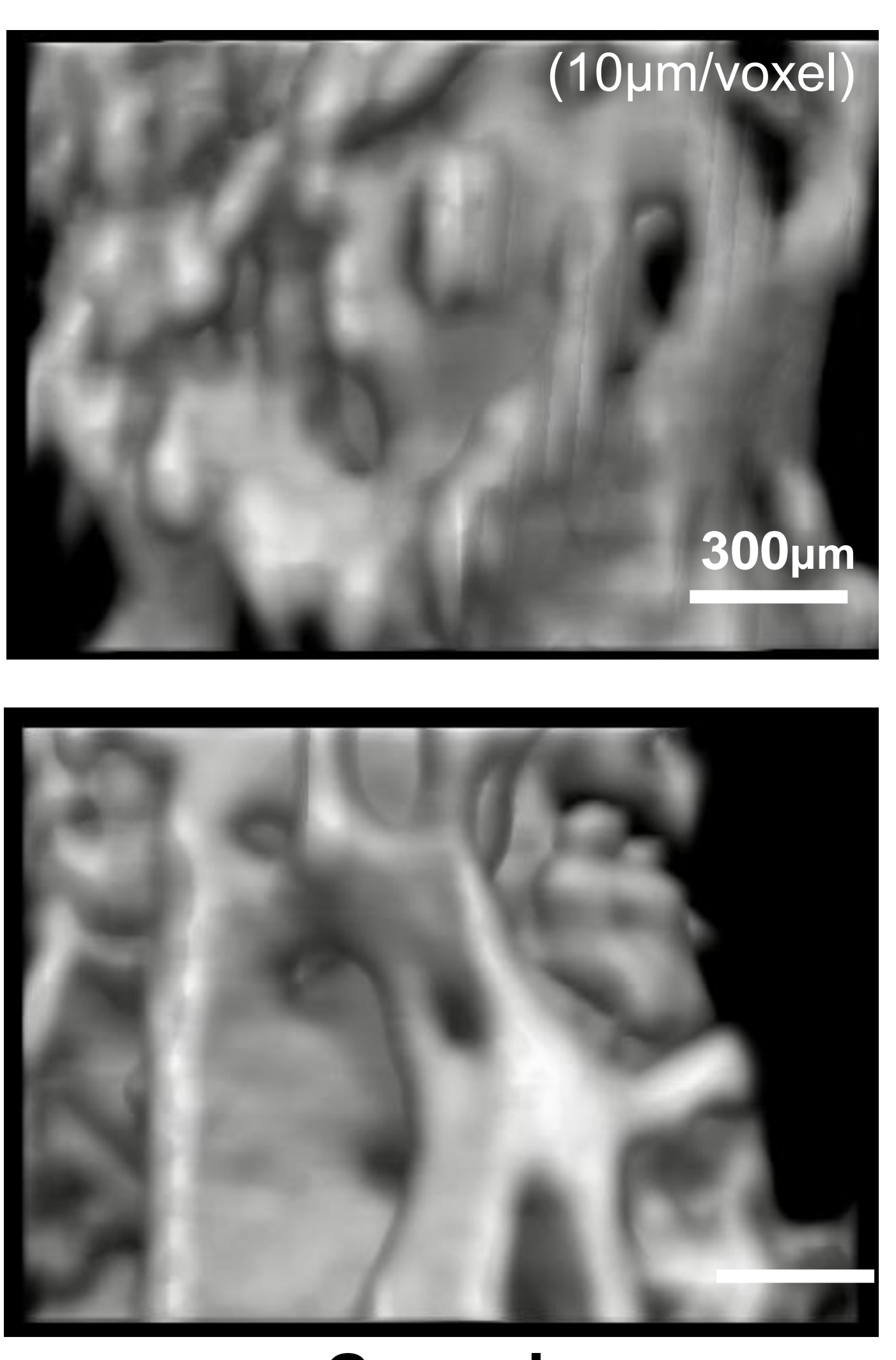

Group L (8wks)
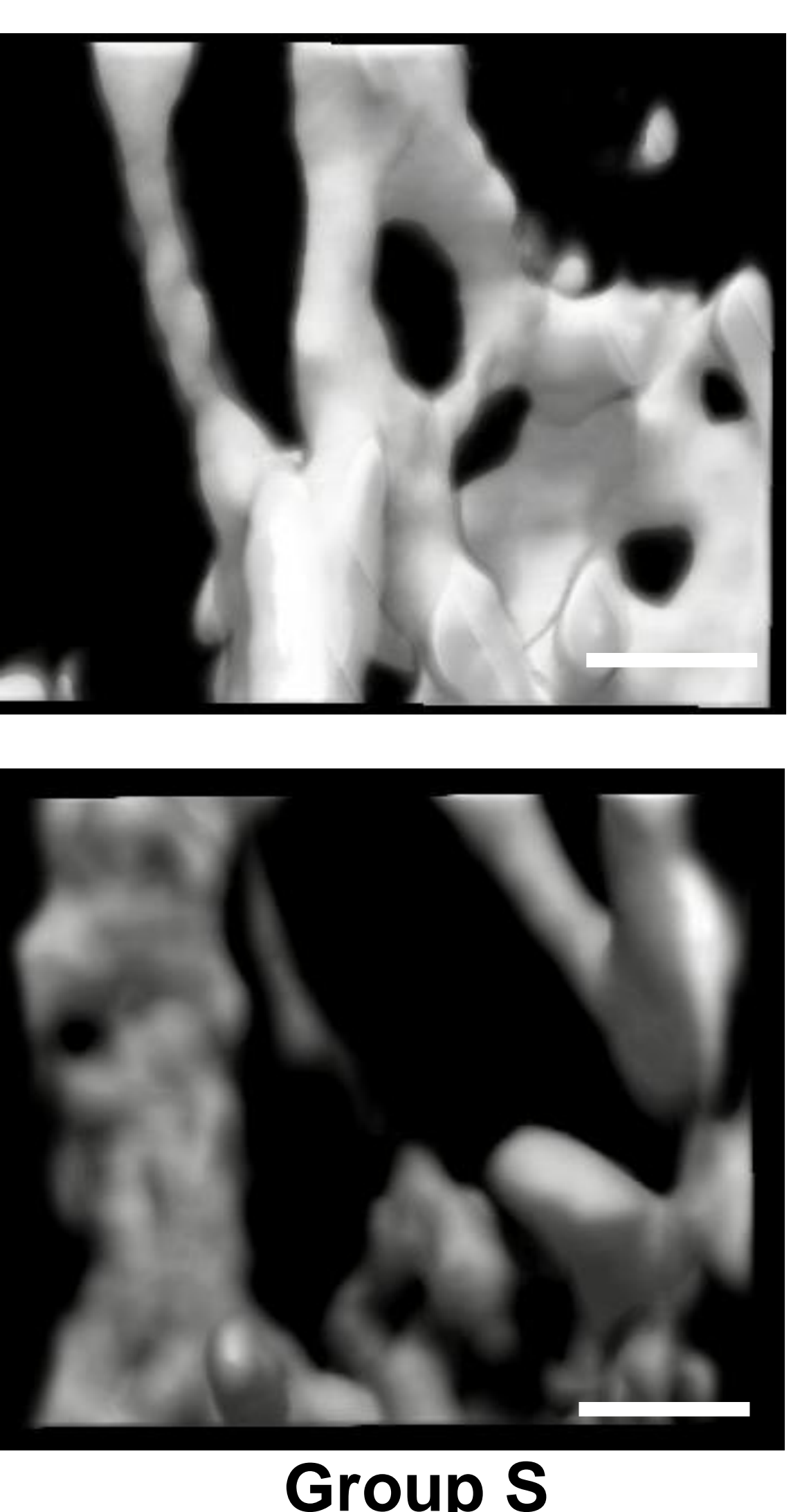

(8wks)

BMD, BV/TV, and other microstructural parameters (Tb.Th BS/BV) significantly increased with time by local ZOL administration

Change of vBMD at Area1

Change of $\mathrm{BV} / \mathrm{TV}$ at Area1

$$
160 \%
$$

$140 \%$

$120 \%$

$100 \%$

$\mathbf{0}$

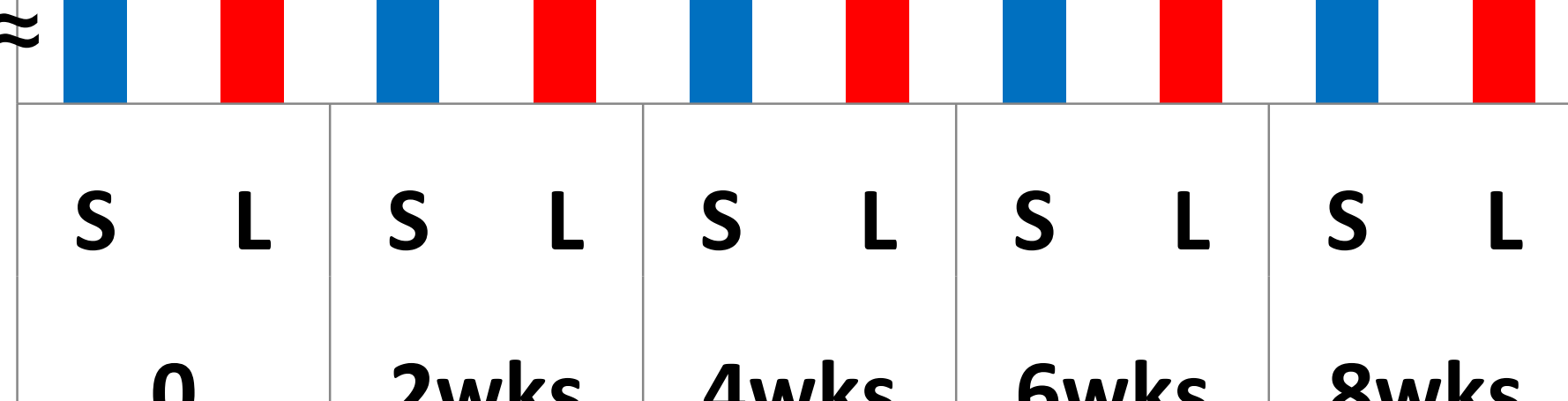

Systemic ZOL administration improved bone loss and deleterious microstructure by OVX, but local ZOL administration had little influence on other bone tissue.

Change of vBMD at Area2

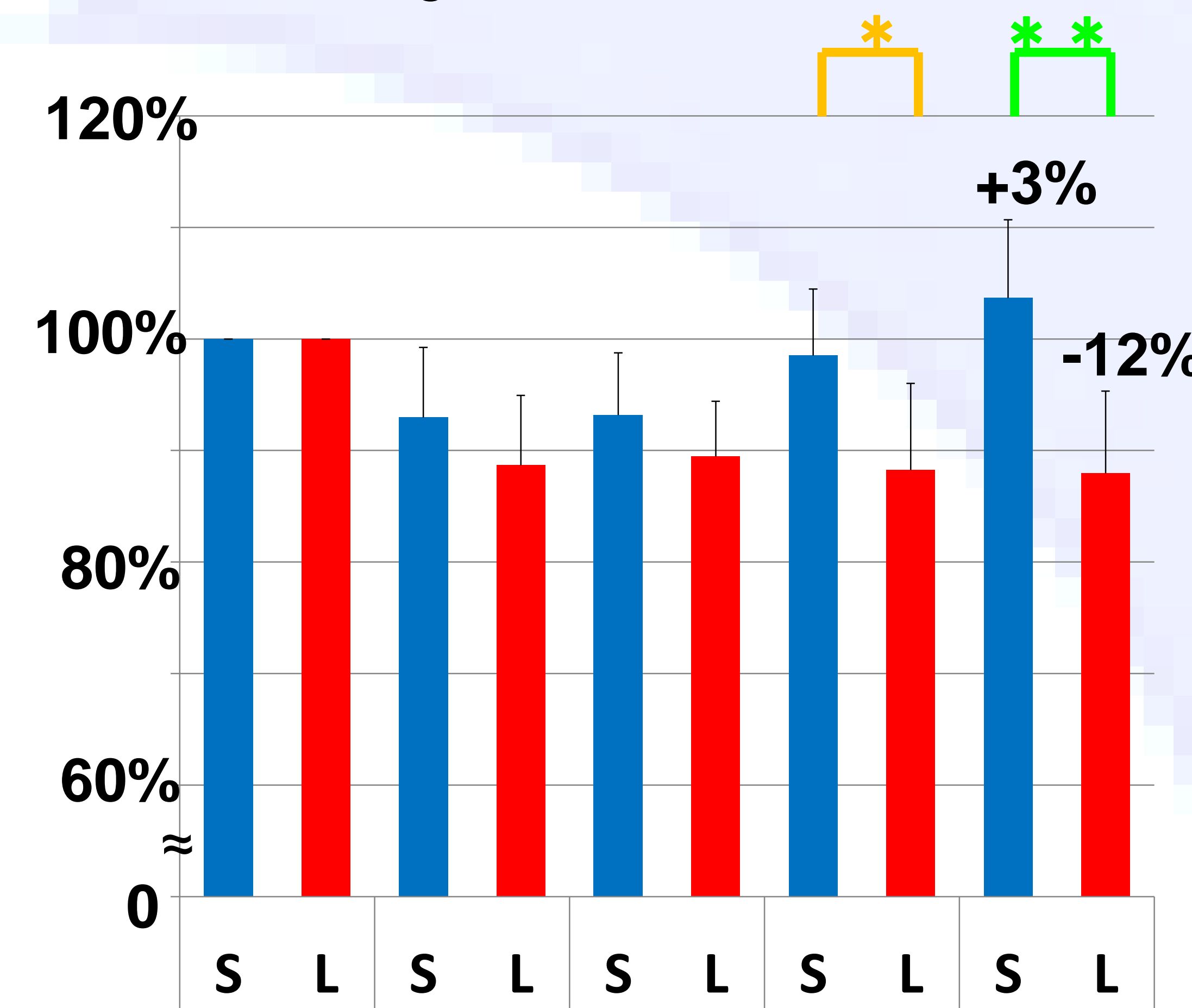

0 2wks $4 w k s$ 6wks $8 w k s$
Change of BV/TV at Area2
$300 \%$

$260 \%$

$220 \%$

$180 \%$

$140 \%$

$100 \%$

$S L S L S L S L S$

0 2wks 4wks 6wks 8wks Student' t test
$120 \%$

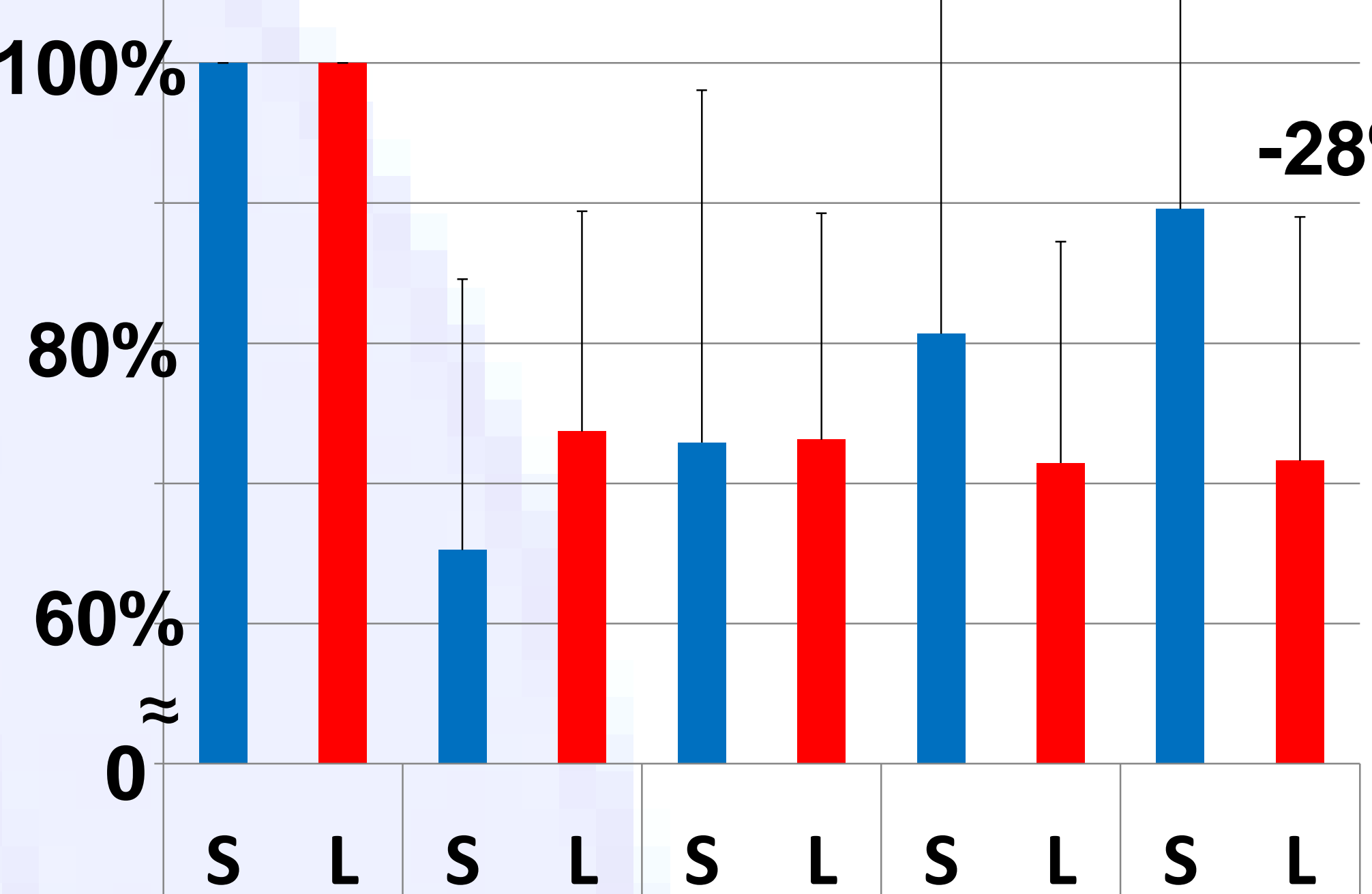

$\begin{array}{lllll}0 & \text { 2wks } 4 w k s \text { 6wks } 8 w k s\end{array}$

Student' t test

\section{Discussions}

There were a few reports that direct administration of $\mathrm{ZOL}$ accelerated fracture healing .But there is no report that direct administration of ZOL improves local osteoporotic lesion.

Bisphosphonates are two key properties

(1) High affinities for bone mineral ( $Z O L>A L N>I B N>R I S)$

(2) Inhibitory effects on osteoclast (ZOL > RIS IBN > ALN)

R.G.G.Russell et al Osteoporos Int (2008)

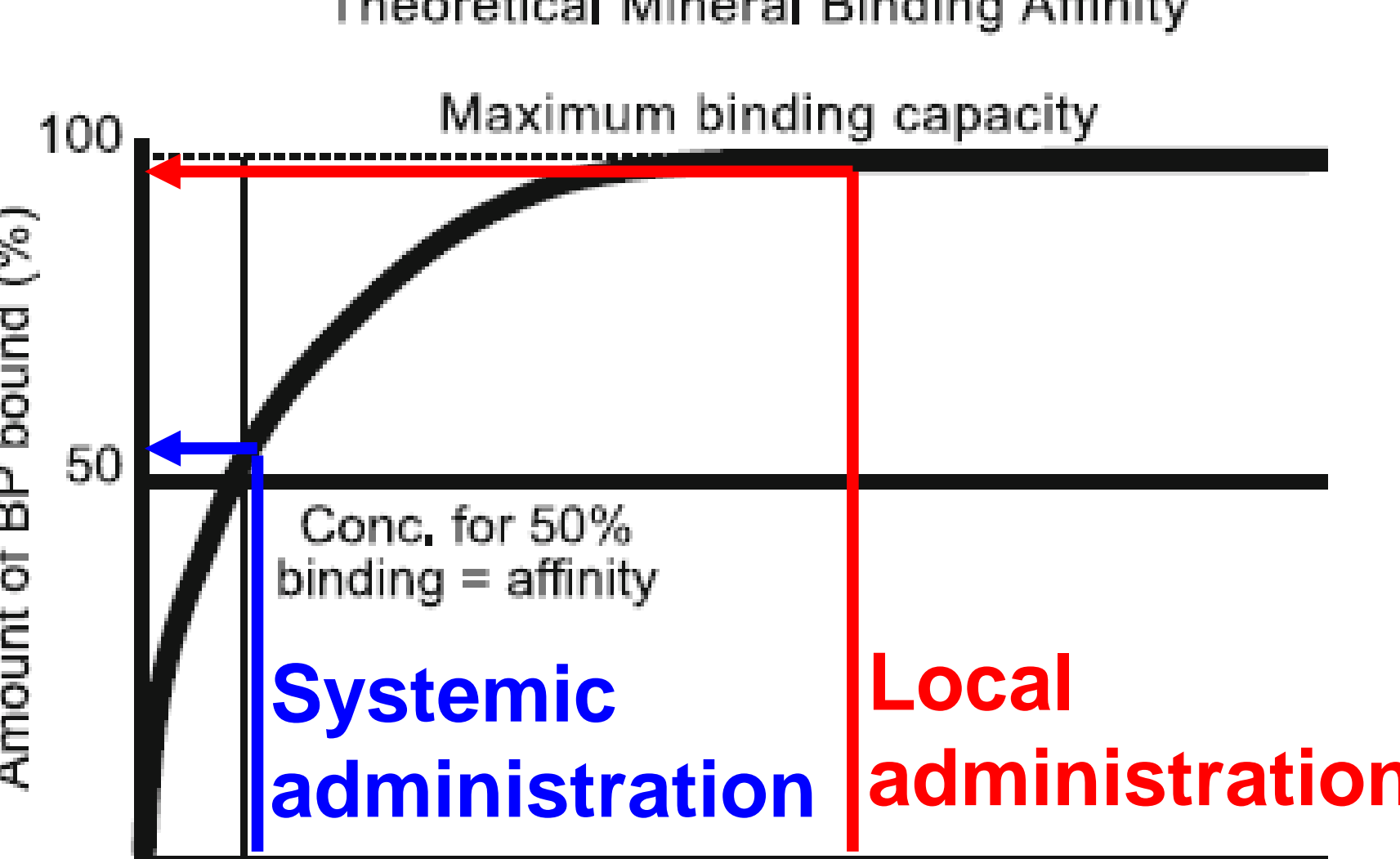

BP concentration in

\section{Future direction}

Further analysis are on going and are planned.

1) Histological analysis of the local lesion (TRAP strain, bone histomorphometrical analysis)

2) Dose testing for local ZOL administration

3) Analysis of effects throughout the body

Biochemical examination, histological examination for other bone tissues...

\section{Conclusions}

Direct administration of high-dose ZOL for local osteoporotic lesions have more beneficial effects on local bone structure than systemic administration, and have little influence on other bone tissue.

\section{Disclosure}

- This work was partially supported by the AO Foundation (Davos, Switzerland) and by the grants from AOSpine Japan.

None of the authors has any financial interest with any of the commercial entities

All authors state that they have no conflicts of interest. 